ORIGINAL ARTICLE

\title{
Health care utilisation of prematurely born, preschool children related to hospitalisation for RSV infection
}

\author{
A Greenough, J Alexander, S Burgess, J Bytham, P A J Chetcuti, J Hagan, W Lenney, S Melville, \\ N J Shaw, J Boorman, S Coles, J Turner
}

Arch Dis Child 2004;89:673-678. doi: 10.1136/adc.2003.036129

See end of article for authors' affiliations

Correspondence to:

Professor A Greenough, Dept of Child Health, King's College Hospital London SE5 9RS, UK; anne.greenough@ kcl.ac.uk

Accepted

18 November 2003

\begin{abstract}
Background: In prematurely born infants with chronic lung disease (CLD), RSV hospitalisation is associated with increased health service utilisation and costs in the first two years after birth.

Aims: To determine whether RSV hospitalisation in the first two years was associated with chronic respiratory morbidity during the preschool years in prematurely born children who had had CLD.

Methods: Retrospective review of readmissions, outpatient attendances, and community care in years 2-4 and, at age 5 years, assessment of the children's respiratory status and their health related quality of life. Comparison was made of the results of children who had had at least one hospitalisation in the first two years after birth for RSV infection (RSV group) to those of the rest of the cohort. Participants were 190 of an original cohort of 235 infants with CLD and a median gestational age 27 (range 22-33) weeks.

Results: The 33 children in the RSV group, compared to the rest of the cohort, had a greater duration of hospital stay and more outpatient appointments. The RSV group had required more prescriptions for all treatments and respiratory medications, and more had used an inhaler. The cost of care of the RSV group was higher (median £2630 [€4000, US\$4800], range £124-18 091 versus £1360 [€2500, US\$3000], range £5-18 929) and their health related quality of life was lower.

Conclusion: In prematurely born children who had developed CLD, RSV hospitalisation in the first two years was associated with chronic respiratory morbidity and increased cost of care.
\end{abstract}

!: both retrospective and prospective studies, respiratory syncytial virus (RSV) infection has been shown to increase the risk of respiratory problems at follow up in previously healthy infants, born at term. ${ }^{12}$ The most compelling evidence is from a prospective study in which 93 matched controls were recruited concurrently with 47 infants who were hospitalised for RSV infection. ${ }^{3}$ The index children, when seen at a mean age of 7.5 years, had a cumulative prevalence of asthma tenfold that of the controls Prematurely born infants, particularly those who have had chronic lung disease (CLD), are more prone to severe RSV infection, as they have small airways, which obstruct from severe RSV oedema and necrotic debris. It seems likely then that RSV infection would also result in chronic respiratory morbidity in prematurely born infants. Surprisingly, that hypothesis has rarely been tested. We, however, showed that, in a cohort of 235 prematurely born children who all had had CLD, the 45 who had been hospitalised with a proven RSV infection subsequently required more frequent and longer hospital admissions, more outpatient attendances, and a greater number of general practitioner consultations for respiratory related disorders in the first two years. ${ }^{4}$ The aim of this study was to determine whether RSV hospitalisation in the first two years was associated with chronic respiratory morbidity, indicated by increased health care utilisation and associated costs and a decreased quality of life during the preschool years in prematurely born children who had had CLD. Such information is important when planning allocation of health service resource and needs to be taken into account when evaluating the impact of preventative strategies.

\section{METHODS}

The study sample consisted of neonates born at less than 32 weeks of gestational age who had been admitted during the first week after birth to one of four neonatal intensive care units between I July 1994 and I July 1997, and subsequently developed CLD (defined as oxygen dependency beyond 28 days after birth). A retrospective review had been made of their care in the community and during any readmission following discharge from the neonatal intensive care unit until the children were 2 years of age. ${ }^{4}$ For the purposes of this study, the parents were reapproached when their child was at least 5 years of age. For those children whose parents gave informed written consent, review was made of their hospital admissions and community care when they were aged 2-4 years inclusive. In addition, parents were asked to complete a respiratory questionnaire and a health related quality of life assessment about their child. The study was approved by the local research ethics committees of each of the four hospitals.

From the neonatal admission, the following data had been retrieved: birth weight, use of antenatal steroids and postnatal surfactant, the development of an airleak (pneumothorax/pulmonary interstitial emphysema) or patent ductus arteriosus (clinical diagnosis with or without echocardiographic confirmation), the duration of ventilatory support, supplementary oxygen, and neonatal unit stay, and the use of high frequency oscillation and/or nitric oxide. ${ }^{4}$ From the general practitioners' records the following data were retrieved: the venue of all hospital readmissions (ICD-10 codes), the number of general practitioner consultations, all medication prescribed, the use of home oxygen, the number of referrals to a health visitor or community paediatric nurse, and the use of community support services

Abbreviations: $C L D$, chronic lung disease; CPAP, continuous positive airway pressure; HDU, high dependency unit; HUI, Health Utility Index; ICU, intensive care unit; IPPV, intermittent positive pressure ventilation; $\mathrm{NICU}$, neonatal intensive care unit; PDA, patent ductus arteriosus; RSV, respiratory syncytial virus 
(for example, physiotherapist, speech therapist, or educational psychologist). For simplicity any member of the primary healthcare team other that the GP and asthma nurse was labelled a specialist. For each hospital admission the following information was recorded: the diagnosis or symptoms leading to the admission, the duration of stay, whether the child was admitted to a paediatric ward, high dependency (HDU) or intensive care unit (ICU), days of supplementary oxygen and intravenous fluids, surgical or therapeutic procedures, and the duration and frequency of all medication. Each child's hospital records were examined to ascertain the number of outpatient attendances. Primary care contacts, hospital admissions, and outpatient attendances were identified as being for respiratory problems by use of ICD codes (ICD-10).

Costs were assessed over the three year period-that is, when the children were aged 2-4 years inclusive. The mean cost of each admission was calculated using data from the National Scheme of Reference Costs (2002). Drug costs were calculated from the British National Formulary prices. The drugs costs included all domestic oxygen therapy costs. The cost of attendance as an outpatient was estimated assuming 15 minutes with a consultant paediatrician and using the mean of the outpatient cost of the four main hospitals. The cost of care by a general practitioner was estimated assuming an 8.4 minute consultation at the surgery ( $£ 18$ per consultation). The cost of a general practitioner's time was based on average net remuneration allowing for capital costs and overheads. The cost of a domiciliary visit by community staff was estimated assuming a 20 minute consultation. The cost of domiciliary visits for health visitors, paediatric nurses, and oxygen nurse specialists was based on average net remuneration for specialist nurses allowing for superannuation, national insurance, travel, and capital overheads ( $£ 27$ per visit). All primary care costs were those reported by Netten and colleagues. ${ }^{5}$ All visits to practice nurses or routine visits to health visitors, for example for immunisations, were not recorded as these were considered the usual costs for children.

When their child was aged 5 years, the parents were sent a respiratory questionnaire to complete to determine whether known risk factors for respiratory morbidity differed between the two groups. The parent(s) was asked to reply to questions regarding parental smoking during pregnancy, their ethnic origin, if the child had been breast fed, whether there was a member of the household who currently smoked, if there was a family history of atopy, the number of long haired pets and siblings under 5 years of age in the house, if the child had been in preschool day care, the frequency and nature of their child's respiratory symptoms, and whether the child had required home oxygen beyond 2 years of age. The child was defined as having a positive family history of atopy if either parent or a sibling suffered from asthma, eczema, or hay fever.

At age 5 years, the children's health related quality of life was assessed using the Health Utilities Index (HUI). The HUI describes a family of generic health status and health related quality of life measures. Parents were sent the HUI 2/3, which incorporates the Mark 2 and Mark 3 HUI questionnaires. The parents were asked to complete the 15 questions to reflect their child's health over the previous four weeks. Health status was determined according to the following attributes: sensation, mobility, emotion, cognition, self-care, pain, vision, hearing, speech, ambulation, and dexterity. Each attribute was measured on a utility scale, such that a score of l was the highest level of function (perfect health) and a score of 0 the lowest function level (death). In order to calculate utility, a scoring algorithm applies utility weights to the respondent's combination of health states. ${ }^{6}$ The HUI 2 has been developed for the interval -0.03 to 1.00 and the HUI 3 for the interval -0.36 to 1.00 ; this extra sensitivity allows states considered worse than death to be picked up as negative scores. A multi-attribute utility score was calculated for each child and used to reflect their health related quality of life.

\section{Analysis}

The infants were divided into two groups according to whether they had had at least one hospital admission with a proven RSV infection (RSV group) or no RSV associated hospitalisation (non-RSV group). RSV infection was considered proven only if the RSV antigen was identified on a nasopharyngeal aspirate. Differences between the two groups were assessed for statistical significance using a KruskalWallis non-parametric analysis of variance, the $\chi^{2}$ test, or Fisher's exact test as appropriate.

To determine whether the RSV group had increased ongoing respiratory morbidity prior to their initial RSV hospitalisation and whether this might explain any increased cost of hospitalisation in the first two years and increased morbidity in years 2-4 inclusive, comparison was made between the RSV proven group and those children who had required at least one hospital admission in the first two years after birth for a respiratory disorder-that is, other than for a RSV proven infection or clinically suspected bronchiolitis (that is, the other respiratory group in Greenough and colleagues ${ }^{4}$.

\section{Patients}

The original study sample had consisted of 235 infants with a median gestational age of 27 weeks (range 22-31) and birth weight of $934 \mathrm{~g}$ (range 510-3000). ${ }^{4}$ Written informed consent for their child to participate in this study was obtained from 190 of the 235 sets of parents; those children did not differ significantly from those who were not recruited (table 1 ). Reasons for failure to recruit were lack of consent $(n=35)$; not registered with a general practitioner $(n=5)$, no contact address $(n=4)$; and child in care $(n=1)$.

\section{RESULTS}

The RSV group with regard to their neonatal admission differed significantly from the non-RSV children only with respect to their duration of NICU admission, although there was also a non-significant trend for them to also have required a longer duration of mechanical ventilation (table 2). At 2-4 years inclusive, the RSV proven group compared to the rest of the cohort required more days in hospital per patient $(\mathrm{p}=0.0436)$ and a greater number of outpatient attendances, both overall $(\mathrm{p}=0.0002)$ and for respiratory problems (mean 2.52 versus $0.85, \mathrm{p}=0.007$ ) (table 3 ). The RSV group also required more prescriptions, both overall $(p=0.004)$ and for respiratory medications $(p=0.0044)$ (table 3$)$. The cost of care was higher in the RSV group $(p=0.0068)$, the cost of care being significantly greater for outpatient attendances, including those for respiratory problems and for prescriptions (table 4). The results of the respiratory questionnaire showed that the RSV proven children did not differ significantly from the rest of the cohort with regard to such risk factors for chronic respiratory morbidity as smoking in the household, day care use, or a family history of atopy, but a greater proportion had required an inhaler $(\mathrm{p}=0.038)$ (table 5$)$. The median HUI 2 multi-attribute utility function was 0.88 (range 0.16-1.00) in the RSV proven children, which was lower than that of the non-RSV children (median 0.95, range $0.03-1.00)(p=0.0088)$. The median HUI 3 multi-attribute score of the two groups did not differ significantly (0.93, range -0.05 to 1.00 versus 0.97 , range -0.32 to 1.00 ). 
Table 1 Comparison of children who were and were not recruited

\begin{tabular}{llll}
\hline & Recruited & Not recruited & $\mathrm{p}$ \\
\hline $\mathrm{n}$ & 190 & 45 & \\
Gestational age (weeks) & $27(23-31)$ & $27(22-31)$ & 0.088 \\
Birth weight (g) & $920(510-3000)$ & $944(632-2190)$ & 0.83 \\
Antenatal steroids & $122(86)$ & $30(86)$ & 0.58 \\
Postnatal surfactant & $163(86)$ & $42(93)$ & 0.17 \\
IPPV & $175(97)$ & $43(98)$ & 1.00 \\
Duration of IPPV (days) & $8(0-103)$ & $8(0-69)$ & 0.99 \\
CPAP & $116(63)$ & $14(32)$ & $<0.001$ \\
PDA & $63(33)$ & $22(49)$ & 0.051 \\
Air leak & $24(13)$ & $11(24)$ & 0.047 \\
Postnatal dexamethasone & $70(38)$ & $19(42)$ & 0.62 \\
Duration of "neonatal" admission (days) & $79(18-827)$ & $91(17-766)$ & 0.43 \\
Home oxygen & $68(36)$ & $20(44)$ & 0.28 \\
\hline Data are presented as the number, median (range), or percentage. & \\
\hline
\end{tabular}

The cost of hospital admission in the RSV proven group prior to their RSV proven admission compared to the cost in those admitted for other respiratory disorders prior to the same postnatal age did not differ significantly (median cost $£ 768$, mean $£ 1068$, range $£ 0-5428$ in the RSV proven group; median cost $£ 768$, mean $£ 1748$, range $£ 0-13580$ in the other respiratory disorders group; $\mathrm{p}=0.09$ ). The costs associated with hospital admissions in the first two years (excluding the neonatal admission and the RSV admission), however, were significantly greater in the RSV group (median cost $£ 3644$, mean 19347 , range $11156-73$ 930) compared to that in the other respiratory disorders group (median cost $£ 2223$, mean $£ 3409$, range £620-23 692) ( $p<0.001)$. Comparison of outcome of those two groups in years $2-4$ inclusive showed that the cost of outpatient attendances differed significantly between them (median $£ 756$, mean $£ 921$, range $£ 44-2745$ in the RSV proven group; median £536, mean £594, range $£ 0$ 2099 in the other respiratory disorders group; $\mathrm{p}=0.0335$ ).

\section{DISCUSSION}

We have shown that health care utilisation and the total cost of care in years 2-4 inclusive was greater in prematurely born children who had had CLD and been hospitalised for RSV infection in the first two years after birth. To our knowledge this is the first study examining whether RSV infection was associated with chronic respiratory morbidity in prematurely born children who had had CLD. Improved survival of very prematurely born infants has resulted in CLD becoming a common outcome of neonatal intensive care. ${ }^{7}$ Our finding that affected infants appear to suffer chronic respiratory morbidity following hospitalisation for RSV infection has important implications for use of health service resources.
We were able to examine 190 of the 235 children recruited into our earlier study examining morbidity in the first two years associated with RSV infection. ${ }^{4}$ Those who were and were not recruited were, however, very similar and only differed significantly with regard to more of those recruited having required continuous positive airway pressure (CPAP) and less having developed an airleak (table 1), thus we do not feel that loss to follow up biased our results. To determine whether hospitalisation for RSV infection in the first two years resulted in chronic respiratory morbidity, we compared those with at least one hospitalisation for a proven RSV infection to the rest of the cohort. We did not include those with a clinical diagnosis of bronchiolitis in the RSV proven group, as we felt a clinical diagnosis was likely to be relatively inaccurate in children with a high rate of underlying respiratory problems. Our hypothesis was supported by the finding that the RSV proven group had a significantly different outcome in the first two years than those with probable bronchiolitis, who fared similarly to those hospitalised for other respiratory problems. ${ }^{4}$ It is possible, however, that our non-RSV group may contain infants who were infected, but RSV was not identified; thus, the comparison we make is likely to be an underestimate of the effect of hospitalisation for RSV infection on chronic morbidity. It was possible that our RSV proven group suffered more chronic morbidity because they were at increased risk prior to their RSV hospitalisation. The similar cost of hospital admissions prior to the RSV proven hospitalisation in the RSV proven group compared to the cost of admissions prior to the same postnatal age in the other respiratory group suggests that this was not the case. The higher cost of hospital admissions during the first two years after birth (excluding the neonatal and RSV admission) in the RSV proven compared to the other

Table 2 Comparison of the antenatal and neonatal characteristics of the RSV proven and non-RSV children

\begin{tabular}{llll}
\hline & RSV proven & Non-RSV & p \\
\hline$n$ & 33 & 157 & \\
Gestational age (weeks) & $28(24-31)$ & $27(23-31)$ & 0.74 \\
Birth weight (g) & $827(640-2178)$ & $940(510-3000)$ & 0.26 \\
Antenatal steroids & $21(81)$ & $101(87)$ & 0.37 \\
Postnatal surfactant & $30(91)$ & $133(85)$ & 0.43 \\
IPPV & $30(97)$ & $145(97)$ & 1.00 \\
Duration of IPPV (days) & $11(0-49)$ & $8(0-103)$ & 0.053 \\
CPAP & $20(63)$ & $96(63)$ & 0.98 \\
PDA & $11(33)$ & $52(33)$ & 1.00 \\
Air leak & $3(9)$ & $21(14)$ & 0.77 \\
Postnatal dexamethasone & $13(40)$ & $57(38)$ & 0.76 \\
Duration of "neonatal" admission (days) & $91(30-360)$ & $76(18-827)$ & 0.015 \\
Home oxygen & $13(39)$ & $55(35)$ & 0.63 \\
\hline Data are presented as the number, median (range), or percentage. & & \\
\hline
\end{tabular}


Table 3 Comparison of readmissions, community care contacts, and treatment requirements of the RSV proven and the non-RSV children

\begin{tabular}{|c|c|c|c|}
\hline & RSV proven & Non-RSV & p value \\
\hline$n$ & 33 & 157 & \\
\hline Hospital admission rate & $1(0-14)$ & $0(0-18)$ & 0.0720 \\
\hline \multicolumn{4}{|c|}{ Days in hospital per patient } \\
\hline Óverall & $1(0-72)$ & $0(0-37)$ & 0.0436 \\
\hline ICU & $0(0-0)$ & $0(0-5)$ & 0.4247 \\
\hline Paediatric ward & $1(0-85)$ & $0(0-36)$ & 0.0712 \\
\hline \multicolumn{4}{|l|}{ Outpatient attendances } \\
\hline Overall & $10(1-39)$ & $5(0-39)$ & 0.0002 \\
\hline Respiratory & $0(0-11)$ & $0(0-7)$ & 0.007 \\
\hline \multicolumn{4}{|l|}{ Specialist visits } \\
\hline Overall & $3(0-12)$ & $2(0-104)$ & 0.4001 \\
\hline Respiratory & $0(0-6)$ & $0(0-7)$ & 0.9124 \\
\hline \multicolumn{4}{|l|}{ GP consultations } \\
\hline Overall & $11(0-51)$ & $10(0-68)$ & 0.2050 \\
\hline Respiratory & $6(0-31)$ & $4(0-55)$ & 0.1907 \\
\hline \multicolumn{4}{|l|}{ Nurse visits } \\
\hline Overall & $0(0-3)$ & $0(0-7)$ & 0.9243 \\
\hline Respiratory & $0(0-3)$ & $0(0-7)$ & 0.1907 \\
\hline \multicolumn{4}{|l|}{ Prescriptions } \\
\hline Overall & $16(0-103)$ & $10(0-140)$ & 0.004 \\
\hline Respiratory & $10(0-32)$ & $4(0-46)$ & 0.0044 \\
\hline
\end{tabular}

respiratory group shows that RSV hospitalisation was associated with increased ongoing morbidity.

Hospitalisation for a proven RSV infection was associated with significantly more outpatient attendances, including for respiratory problems and a requirement for significantly more prescriptions (table 3 ). It is possible that the paediatricians were influenced by the knowledge that children who they were following had had an RSV hospitalisation and, as a consequence, offered a greater number of follow up appointments. The possibility of a link between RSV hospitalisation in preterm infants who had BPD with ongoing morbidity, however, has rarely been investigated, so such a policy would have been surprising; in addition, the RSV hospitalisation did not always occur at the "follow up" hospital. It seems, therefore, more likely that the extra outpatient appointments were given because the children who had had an RSV hospitalisation had ongoing morbidity. In addition, there was also a significant difference in the duration of hospital admission between the two groups and, although in both this was short, the duration of hospital admission seems unlikely to have been influenced by a past history of RSV hospitalisation. There was no significant difference in the number of primary care contacts between the two groups, but this is likely to have been influenced by the greater number of outpatient attendances in the RSV proven children. The greater outpatient attendance and requirement for prescriptions resulted in a cost of care which was twice as great for the RSV proven children compared to the rest of the cohort (table 4).

The RSV proven children differed significantly from the other children with regard to having required a longer duration of neonatal unit stay and a tendency for a longer duration of mechanical ventilation (table 2). It might then be suggested that the RSV proven children had a greater severity of initial illness and hence suffered greater chronic respiratory morbidity. There was, however, no statistically significant differences in the proportions of the two groups who had developed a patent ductus arteriosus (PDA) or an airleak, which are associated with severe neonatal respiratory disease, nor was there a greater requirement for postnatal dexamethasone or home oxygen in the RSV proven group (table 2). The results of the respiratory questionnaire (table 5) show that the two groups were similar with respect to other risk factors for respiratory morbidity at follow up; in particular, similar proportions had been exposed to smoking antenatally and currently, had a family history of atopy, were exposed to long haired pets, or had attended day care, and their parents were home owners (as a marker of social class). Although we were unable to validate the results of the questionnaire by examination of the neonatal records as the relevant information was rarely documented within them, the same questionnaire was applied to all of the children. Thus, the lack of difference in risk factors for respiratory morbidity highlighted by the results of the respiratory

Table 4 Comparison of the cost of care (in pounds sterling) of the RSV proven and the non-RSV children

\begin{tabular}{lllr}
\hline & RSV proven & Non-RSV & p value \\
\hline $\mathrm{n}$ & 33 & 157 & \\
Hospital admissions & $526(0-12592)$ & $9(0-14399)$ & 0.0799 \\
$\begin{array}{l}\text { Outpatient attendances } \\
\text { Overall }\end{array}$ & $756(44-2745)$ & $356(0-3931)$ & $<0.0001$ \\
$\quad$ Respiratory & $0(0-979)$ & $0(0-623)$ & 0.0076 \\
Prescriptions & $40(0-8513)$ & $27(0-5170)$ & 0.0019 \\
$\quad$ Overall & $0(0-0)$ & $0(0-68)$ & 0.6466 \\
Home oxygen & $593(15-6600)$ & $329(0-7919)$ & 0.2441 \\
Total primary care & $2630(125-18092)$ & $1361(5-18929)$ & 0.0154 \\
Total cost & & \\
\hline Data are presented as the number or the median (range). & &
\end{tabular}


Table 5 Comparison of the results of the respiratory questionnaires of the RSV proven and the non-RSV children.

\begin{tabular}{lrrl}
\hline & RSV proven & Non-RSV & p \\
\hline Smoking & & & \\
$\quad$ In pregnancy & $9(28)$ & $38(26)$ & 0.76 \\
$\quad$ Currently & $14(22)$ & $67(22)$ & 0.97 \\
Family history of atopy & $24(75)$ & $91(59)$ & 0.09 \\
Attendance at day care & $1(17)$ & $3(14)$ & 0.36 \\
Long haired pets & $5(16)$ & $35(24)$ & 0.72 \\
Parents home owners & $21(66)$ & $105(71)$ & 0.11 \\
Home oxygen beyond two years & $3(9)$ & $4(3)$ & 0.24 \\
Cough & $23(74)$ & $92(63)$ & 0.92 \\
$\quad$ Ever & $9(29)$ & $41(28)$ & 0.49 \\
$\quad$ More than once a week & $13(42)$ & $53(35)$ & 0.97 \\
Wheeze & $2(7)$ & $10(7)$ & 0.038 \\
$\quad$ Ever & $27(82)$ & $99(63)$ & 0.29 \\
$\quad$ More than once a week & $15(46)$ & $56(36)$ & \\
Used an inhaler & & & \\
Diagnosis of asthma & &
\end{tabular}

questionnaire, we feel emphasises that the differences shown between the two groups with regard to chronic respiratory morbidity is a reflection of hospitalisation for RSV infection.

RSV infection in children born at term has been associated with an increase in asthma. ${ }^{13}$ The parents in this study did not report that the children who had been hospitalised for RSV infection had been more likely to have been diagnosed as having asthma. It is possible that practitioners may have been unwilling to make a diagnosis of asthma in these children, because of their relatively young age, and also may have ascribed any respiratory symptoms to the children having suffered CLD. The RSV proven children had required significantly more prescriptions and the increased treatment in that group may explain why the parents did not report that their children were more likely to wheeze or cough frequently. The RSV proven children required significantly more outpatient attendances for respiratory problems and were more likely to have required an inhaler. In children born at term, RSV infection is associated with a greater risk of asthma at follow up and bronchodilator usage. ${ }^{1}$ We suggest that the greater use of inhalers in the RSV proven group indicates they too were more likely to have asthma. To test that hypothesis, it is our intention to re-examine these children when they are sufficiently old enough to undertake pulmonary function tests.

The median HUI scores were lower in children who had been hospitalised for RSV infection. The Mark 2/3 HUI 2/3 is a common instrument for measuring health state preferences. The HUI 2 measures seven attributes of health status describing 24000 unique health states, while the extended Mark 3 system describes 972000 unique health states. The HUI 2 was originally developed for paediatric application and clinical evaluation studies, whereas HUI 3 was developed for use in adults and population surveys; this may explain why differences we show between the two groups only reached statistical significance for the HUI 2. The intent of the HUI is to document the extent to which deficits in health status for each attribute inhibit or prohibit normal functioning rather than to report the level at which an individual chooses to function (performance). The precision of the health state descriptions in the HUI 2 system is derived from the large number of combinations of levels among the attributes which provides a framework for capturing important changes in health status over time. Unlike alternative methods of deriving utility such as the EuroQoL, the HUI system has been validated for both paediatric and adult application. The scoring algorithm for the HUI was first developed from interviews with the parents of childhood cancer patients and parents of similarly aged schoolchildren. ${ }^{8}$ Nevertheless, there are caveats in assessing health related quality of care of children. Application of HUI 2 to a small population of child survivors of brain tumours showed that there were differences between the judgements of the clinicians, nurses, and parents regarding the children's health status, clinicians reporting a lower morbidity. ${ }^{9}$ It is also important to remember that parental reports represent a proxy response for the children's function. Although parents are clearly the most knowledgeable source of information about their children, some attributes in a multi-attribute health status measure such as HUI are more difficult to observe than others. For example, although parents may observe directly whether a child requires help with mobility, their judgements about pain or emotion are based on interpretation of their child's behaviour and thus provide only indirect accounts. Nevertheless, in this study parents completed the HUI for all children, thus there was no bias between the two groups and yet a statistically significant difference was seen between them. These results, then, suggest that prematurely born children who are hospitalised for RSV infection in the first two years have a poorer health related quality of life at 5 years of age.

In summary, the children who had been hospitalised with a proven RSV infection in the first two years required more health care for respiratory problems in years 2-4 inclusive; in addition, their parents reported them to have a poorer health related quality of life. We therefore conclude that RSV infection results in significant chronic respiratory morbidity in children born prematurely who had had CLD.

\section{ACKNOWLEDGEMENTS}

The research nurses were funded by Abbott Laboratories Ltd. We are grateful to Ashley Cain and Patricia Griffiths for secretarial assistance.

\section{Authors' affiliations}

A Greenough, J Bytham, King's College Hospital, London, UK J Alexander, W Lenney, S Melville, North Staffordshire Hospital, Stokeon-Trent, UK

S Burgess, P A J Chetcuti, Leeds General Infirmary, UK

J Hagan, N J Shaw, Liverpool Women's Hospital, UK

J Boorman, S Coles, F Pang, Abbott Laboratories Ltd, Maidenhead, UK

J Turner, Therapeutic Management, Crowthorne, UK

\section{REFERENCES}

1 Noble V, Murray M, Webb MSC, et al. Respiratory status and allergy nine to ten years after acute bronchiolitis. Arch Dis Child 1997;76:315-19. 
2 Pullen CR, Hey EN. Wheezing, asthma and pulmonary dysfunction 10 years after infection with respiratory syncytial virus in infancy. BMJ 1982;284: 1665-9.

3 Sigurs N, Bjarnson R, Sigurbergsson F, et al. Respiratory syncytial virus bronchiolitis in infancy is an important risk factor for asthma and allergy at age seven. Am J Respir Crit Care Med 2000;161:1501-7.

4 Greenough A, Cox S, Alexander J, et al. Health care utilisation of infants with chronic lung disease, related to hospitalisation for RSV infection. Arch Dis Child 2001 :85:463-8.

5 Netten A, Dennett J, Knight J. Unit costs of health and social care. University of Kent, Personal Social Services Research Unit, 2000
6 Torrance GW, Feeny DH, Furlong WJ, et al. Multiattribute utility function for a comprehensive health status classification health system. Health Utility Index Mark 2 Medical Care 1996;34:702-22.

7 Johnson AH, Peacock JL, Greenough A, et al. High frequency oscillatory ventilation for the prevention of chronic lung disease of prematurity. N Engl J Med 2002;347:633-42.

8 Cadman D, Torrance GW, Taylor DW. Measurement of quality of life in endstage renal disease: the trade off approach. New York: Oxford Press, 1987:1-182.

9 Barr RD, Pai MKR, Weitzman S. A multi-attribute approach to health status measurement and clinical management-illustrated by an application to brain tumours in childhood. Int J Oncol 1994;4:639-48.

\section{IMAGES IN PAEDIATRICS}

\section{A swollen shoulder}

S

wellings of this size are unusual in UK clinical practice

This 15 year old boy presented with a swollen left shoulder that had grown slowly over the course of a year, with increasing restriction of movement. On examination, he was malnourished and anaemic. Plain radiographs and magnetic resonance imaging showed a destructive lesion in the left scapula extending into the chest wall. Bone scan and computed tomography of the chest showed no metastases. Histology confirmed a diagnosis of primitive neuroectodermal tumour/Ewing's sarcoma. He was given two cycles of chemotherapy which significantly decreased the tumour mass in preparation for (possible) surgery. Delay between first symptoms and diagnosis has been reported to be common in Ewing's sarcoma, ${ }^{1}$ but we feel this boy's case to be unusual.

H J Tan, S Power, S Hodgson Departments of Paediatrics and Surgery, Royal Bolton Hospital Lancashire, UK; huijeentan@hotmail.com

A Abudu Orthopaedic Oncology Service, Royal Orthopaedic Hospital

Birmingham, UK doi: 10.1136/adc.2003.042796
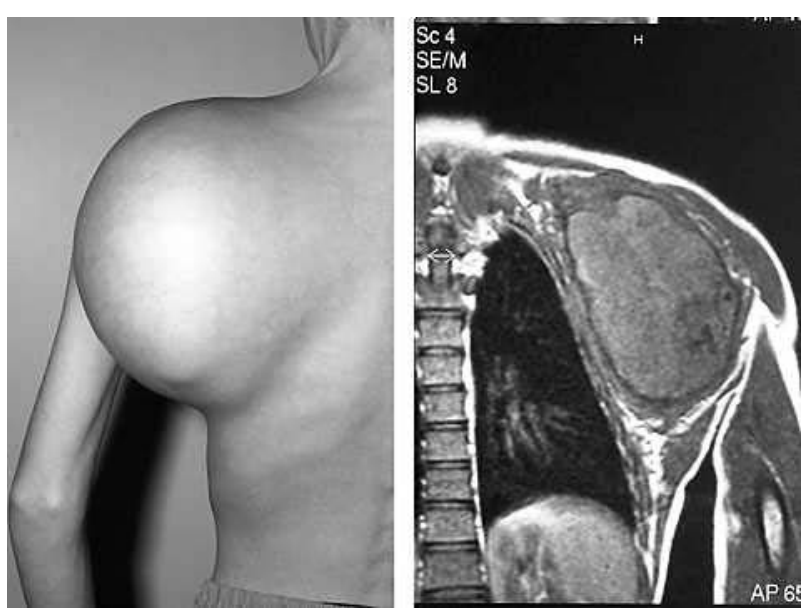

\section{Reference}

1 Grier HE. The Ewing family of tumors. Pediatr Clin North Am 1997;44:991-1004. 